\title{
Existence and Predication in Free Logics
}

\author{
Guilherme Kubiszeski
}

Secretaria de Estado de Educação do Distrito Federal, Brasil

email:guilhermefk4@gmail.com

\begin{abstract}
:
This paper presents a fundamental difference between negative semantics for free logics and positive ones regarding the logical relations between existence and predication. We conclude that this difference is the key to understand why negative free logics are stronger, i.e., they prove more, than positive free logics.

Keywords: free logics, existence, predication, square of opposition.
\end{abstract}

\section{Introduction}

Classical first-order logic assumes that all singular terms in the domain of quantification denote. Free logics do not make such an assumption, as they allow the use of empty singular terms. This means that in free logics one can assign a truth-value not only to non-empty-termed atomic formulae, but also to empty-termed ones. The way that assignment is done is the precise difference between positive free logics and negative free logics. While in the latter all empty-termed atomic formulae are false, the former allow some of them to be true [4]. Therefore, the distinction is mainly semantic. It is important to note that "free" is a shorthand for "free of ontological commitment" regarding singular terms in a formal language. This means that not all singular terms in a free semantics must have an interpretation in a specific domain of "existing" objects. The emphasis on the formal character of the language is needed, since we do not want to consider Russell, for instance, a free logician. In other words, the acceptance of free singular terms in a natural language does not make one a proponent of free logics.

A well-known inconvenient surrounding classical predicate logic is its inability to translate using only existential quantification, individual terms and identity - sentences like "Socrates does not exist" without commitment to the existence of Socrates. The most intuitive way of translating the corresponding affirmative sentence is by means of the formula ' $\exists x(x=s)$ ' (informally: "there is an $x$ such that $x$ is identical to Socrates"), which happens to be a valid formula in classical firstorder systems, as every singular term must denote to make the sentence true - assuming, of course, that ' $s$ ' is an element of the interpreted language. Since ' $\sim \exists x(x=s)$ ' is false under all interpretations, those systems fail to provide an intuitive translation of the negative existential statements of everyday talk, such as "Sherlock Holmes does not exist" or "Santa Claus does not exist". Of course, this incapacity does not rely upon a syntactic impossibility to symbolize non-empty terms, but rather on an assumed ontological commitment with every bound variable. Surely, there are syntactic 
formulations of sentences including empty terms in non-free logics (see [5] and [6]), but such formulations resort to more than just existential quantification, individual terms and identity.

On the other hand, free logics accommodate that kind of negative existential statements. Usually, free logics employ a one-place predicate ' $E$ !', such that ' $E ! s$ ' is true if and only if ' $s$ ' is non-empty. 'E!' may be defined as follows:

$$
E ! t={ }_{d f} \exists x(x=t)
$$

If a term ' $s$ ' is non-denotative, ' $\sim E ! s$ ' (informally: " $s$ does not exist") turns out true. This is the common ground between positive and negative free semantics: a formula asserting the existence of some object may be true or false depending on whether its singular term denotes. But when it comes to assign a truth value to atomic formulae of the form ' $P t_{1} \ldots t_{n}$ ', where ' $P$ ' is an $n$-place predicate other than ' $E$ !' and ' $t_{1}$ '... ' $t_{n}$ ' are singular terms, the situation changes. Positive free logics allow some formulae of the form ' $P t_{1} \ldots t_{n}$ ' to be true even when their singular term is empty; negative free logics, au contraire, assign the truth-value false to all formulae of the form ' $P t_{1} \ldots t_{n}$ ' when their singular term is empty. One could ask to what extent those negative semantics are properly free, since they do not seem to be suitable for treating some sentences containing empty singular names (for instance, fiction talk). However, it is important to note that they still allow individual constants and variables without denotation, and this is precisely what makes a logic "free". Let us consider the semantical clauses for ' $E ! t$ ' and ' $P t_{1} \ldots t_{n}$ ' in both families of free logics.

\section{Semantics for Free Logics}

Assume a usual first-order language plus ' $E$ !' and call it $L$. Let $\langle D, I\rangle$ be a pair where $D$ is a possibly empty set and $I$ an interpretation function such that: (1) for each individual constant $t$ of $L$, either $I(t) \in D$ or $t$ is undefined, and (2) for each $n$-place predicate $P$ of $L, I(P) \subseteq D^{n}$. The pair $\langle D, I\rangle$ is a negative model for $L$. A valuation function $V$ is a function from the set of formulae of $L$ to the set $\{\mathrm{T}, \mathrm{F}\}$ of truth-values. Formulae of the form ' $P t_{1} \ldots t_{n}$ ' and ' $E !{ }^{\prime}$ ' are evaluated as follows [4]:

- $V\left(P t_{1} \ldots t_{n}\right)=\mathrm{T}$ if and only if $I\left(t_{1}\right), \ldots, I\left(t_{n}\right) \in D$ and $\left\langle I\left(t_{1}\right), \ldots, I\left(t_{n}\right)\right\rangle \in I(P)$.

- $V(E ! t)=\mathrm{T}$ if and only if $I(t) \in D$.

Informally, the first clause says that a predication or a relational sentence may be true only if its terms denote. So "Sherlock Holmes is a detective" and "Santa Claus wears a beard" turn out false. The second only says that an existential sentence like "Socrates exists" is true only if the term "Socrates" is non-empty.

Positive semantics, on the other hand, are often split-domain semantics. Assume the same first-order language $L$. Then take a domain and split it into two: $D_{i}$ (the inner domain, possibly empty) and $D_{o}$ (the outer domain, necessarily non-empty). ${ }^{1} I$ is an interpretative function such that for each individual constant $t$ of $L$, either $I(t) \in D_{i}$ or $I(t) \in D_{o}$. The triple $<D_{i}$, $D_{o}, I>$ is a positive model for $L$. A valuation function $V$ is a function from the set of formulae of $L$ to the set $\{\mathrm{T}, \mathrm{F}\}$ of truth-values. Formulae of the form ' $P t_{1} \ldots t_{n}$ ' and ' $E ! t$ ' are evaluated as follows [4]:

- $V\left(P t_{1} \ldots t_{n}\right)=\mathrm{T}$ if and only if $\left\langle I\left(t_{1}\right), \ldots, I\left(t_{n}\right)\right\rangle \in I(P)$.

- $V(E ! t)=\mathrm{T}$ if and only if $I(t) \in D_{i}$. 
So positive free logics have a weaker clause for the truth of formulae of the form ' $P t_{1} \ldots t_{n}$ ' than negative ones, since they can be true even if none of their terms refer. The second clause, informally read, simply means that existence is the same as being in the inner domain. Informally put, "Sherlock Holmes is a detective" and "Santa Claus wears a beard" may turn out true according to positive semantics. Members of the outer domain, understood as "nonexisting" objects, may belong to the interpretation of one-place predicates, as well as be an element of $n$-tuples that form the interpretation of $n$-place predicates (for $n>1$ ).

\section{Lambert's Square}

Lambert [3, p. 135] argued that the classical principles of Specification and Particularization give rise to a square of opposition built not only out of quantified formulae, but out of nonquantified ones as well. It looks like this:

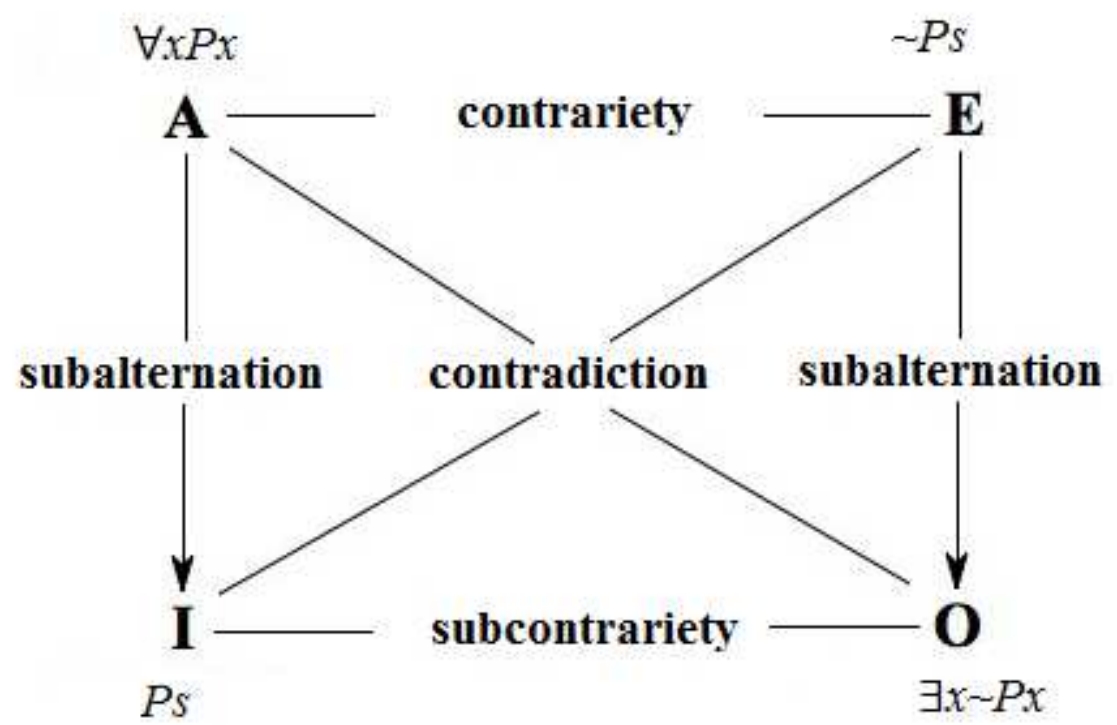

Lambert correctly showed that the inferences from $\forall x P x$ to $P s$ and from $\sim P s$ to $\exists x \sim P x$ only works with an important presupposition - that ' $s$ ' denotes, or equivalently, that ' $\exists x(x=s)$ ' is true. He chose to consider the classical version of both principles simply wrong and to weaken them as follows:

- Specification $\forall x P x \rightarrow(E ! s \rightarrow P s)$

- Particularization: $P s \rightarrow(E ! s \rightarrow \exists x P x)$

Lambert's square sets positive and negative free logics equally apart from classical predicate logic, as both deny the classical version of Specification and Particularization, substituting them by the weaker versions presented above. When it comes to the logical relations between quantified formulae and its corresponding instantiations, positive and negative free logics are closer to each other than to classical first-order systems.

\section{The Categories Square}

In Aristotle's treatise Categories [1], there is an implicit square different from the most-known diagram implicit in On Interpretation:

'Socrates is ill' is the contrary of 'Socrates is well'. Yet we cannot maintain even here that one statement must always be true and the other must always be false. For, if Socrates really 
exists, one is true and the other is false. But if Socrates does not exist, both the one and the other are false. To say 'he is ill' will be false, and to say 'he is well' will be false, if no Socrates so much as exists. [...] To return to affirmation and negation. Of these we may say in all cases that one must be false and one true, be the subject existent or not. For, if Socrates really exists, 'he is ill' or 'not ill' must be true; 'he is ill' or 'not ill' must be false. And the same, if he does not exist. For, provided he does not exist, it is false to pronounce 'he is ill'; 'he is not ill', however, is true [13b20-33].

This passage makes clear Aristotle's position on empty terms: any affirmation (affirmative predication, of the form "S is $\mathrm{P}$ " or "S is not-P") with a non-referring term is false, for its sentential negation (negative predication, of the form "S is not $\mathrm{P}$ ") will be true. By contraposition on "if $s$ does not exist, then $s$ is not $P$ ", we get "if $s$ is $P$, then $s$ exists". The resemblance with negative free semantics is clear. The following square is implicit in those lines:

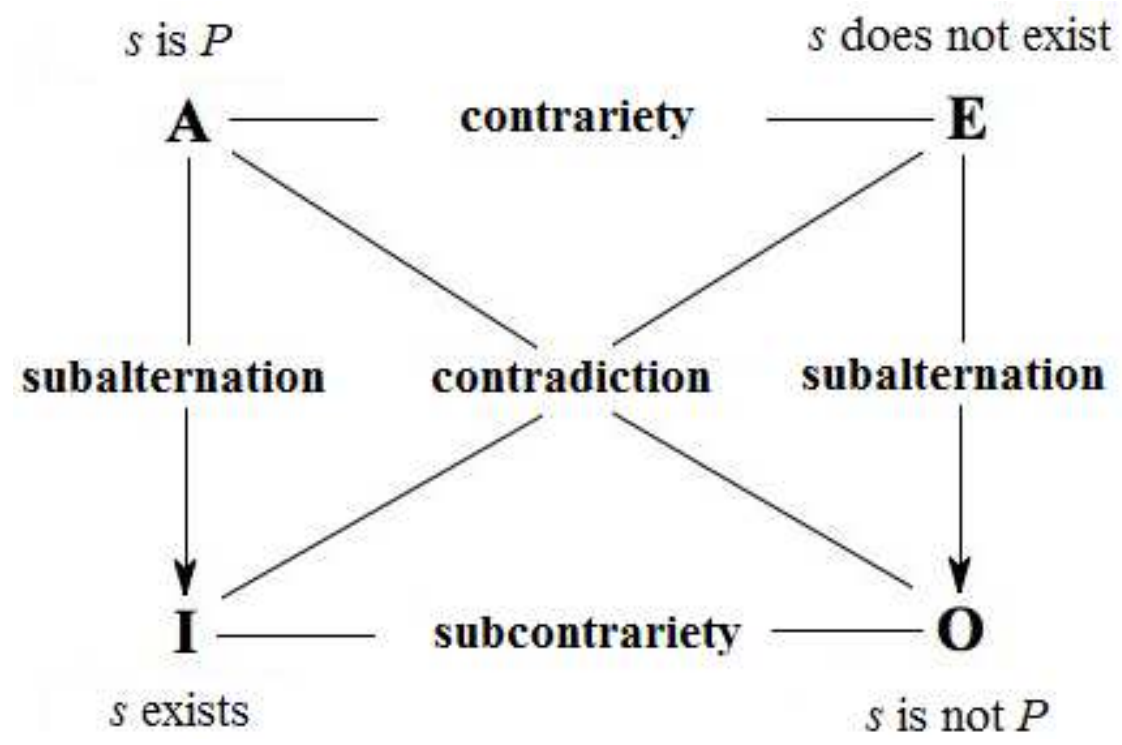

Those logical relations between predicative and existential sentences allow us to say that Aristotle was a forerunner of negative free logics.

Once couched in first-order language, the implicit square of the Categories looks like this:

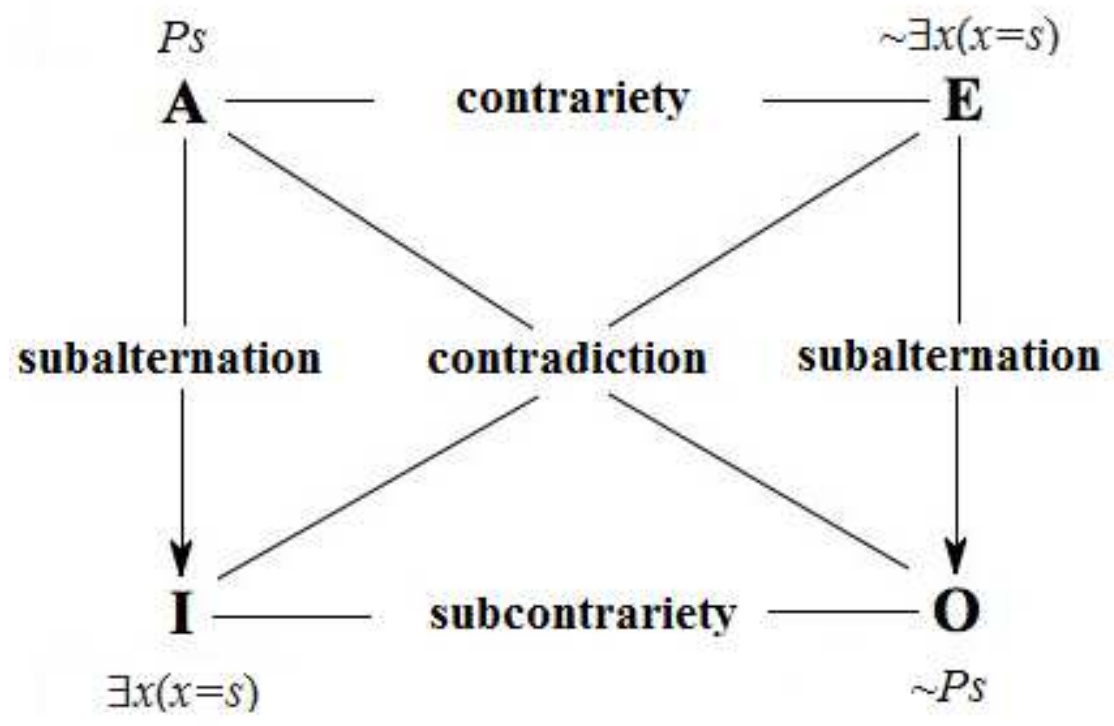


None of those relations besides contradiction is valid in positive free logic. A positive free model which falsifies contrariety, subcontrariety and subalternation can be easily constructed ${ }^{2}$ :

$$
\begin{gathered}
D_{o}=\{\mathrm{s}\} \\
D_{i}=\{\} \\
I(P)=\{s\}
\end{gathered}
$$

According to the clauses for ' $P t_{1} \ldots t_{n}$ ' and ' $E$ ! $t$ ' in positive semantics, we have $V(P s)=\mathrm{T}$ and $V(E ! s)=\mathrm{F}$. As $E ! t={ }_{d f} \exists x(x=t)$, we also get $V(\exists x(x=s))=\mathrm{F}$. So a positive free logic does not represent Aristotle's stance on sentences containing empty singular terms.

A negative free semantics, on the other hand, handles perfectly the semantical ideas expressed in the Categories passage. All of the following are valid formulae in negative free $\operatorname{logics}^{3}$ :

$$
\begin{gathered}
P s \mid \sim \exists x(x=s) \\
\exists x(x=s) \vee \sim P s \\
P s \rightarrow \exists x(x=s) \\
\sim \exists x(x=s) \rightarrow \sim P s
\end{gathered}
$$

The proofs are trivial, and can be carried out by reductio ad absurdum. Assume that $V$ (Ps $\sim \exists x(x=s))=\mathrm{F}$; then, $V(P s)=V(\sim \exists x(x=s))=\mathrm{T}$. According to the clause for ' $P t_{1} \ldots t_{n}$ ' in negative semantics, if $V(\sim \exists x(x=s))=\mathrm{T}$, then $V(P s)=\mathrm{F}$, which contradicts $V(P s)=\mathrm{V}$. So $P s \mid \sim \exists x(x=s)$ is a valid formula in negative free logic.

Assume that $V(\exists x(x=s) \vee \sim P s)=\mathrm{F}$. Then $V(\exists x(x=s))=V(\sim P s)=\mathrm{F}$. From the latter result we have $V(\sim \exists x(x=s))=\mathrm{T}$ and $V(P s)=\mathrm{T}$, which is impossible under the clause for ' $P t_{1} \ldots t_{n}$ ' in negative semantics. So $\exists x(x=s) \vee \sim P s$ is a valid formula in negative free logic.

Assume that $V(P s \rightarrow \exists x(x=s))=\mathrm{F}$. Then we have $V(P s)=\mathrm{T}$ and $V(\exists x(x=s))=\mathrm{F}$. If $V(P s)=$ $\mathrm{T}$, then we have, according to the clause for ' $P t_{1} \ldots t_{n}$ ' in negative semantics, $V(\exists x(x=s))=\mathrm{T}$, which contradicts $V(\exists x(x=s))=\mathrm{F}$. So $P s \rightarrow \exists x(x=s)$ is a valid formula in negative free logic.

Assume that $V(\sim \exists x(x=s) \rightarrow \sim P s)=\mathrm{F}$. Then we have $V(\sim \exists x(x=s))=\mathrm{T}$ and $V(\sim P s)=\mathrm{F}$. From this result we have $V(P s)=\mathrm{V}$ and $V\left((\exists x(x=s))=\mathrm{F}\right.$. According to the clause for ' $P t_{1} \ldots t_{n}$ ' in negative semantics, if $V(P s)=\mathrm{T}$, then $V(\exists x(x=s))=\mathrm{T}$, which contradicts $V((\exists x(x=s))=\mathrm{F})$. So $\sim \exists x(x=s) \rightarrow \sim P s$ is a valid formula in negative free logic.

Even though classical predicate systems are set apart from free logics in the context of Lambert's square, in the square drawn from Aristotle's Categories the situation is quite different. We can easily see that the latter is satisfied not only by negative free logics, but also by classical predicate logic. As ' $\exists x(x=s)$ ' is true in all classical models, ' $\exists x(x=s)$ ' turns out false; it is clear from these values that all first-order formulae corresponding to the relations expressed in the square are valid in classical logic. A conclusion immediately suggests itself: when it comes to consider the logical relations between atomic and existential formulae (and their corresponding negations), classical first-order logic is closer to negative free logics than to positive ones.

\section{Conclusion}

To sum up, there is a fundamental sense in which negative free logics - rather than positive free ones - more closely resemble classical predicate logic. The Categories square, once understood in the context of modern logic, appears as an important structure to show that families of positive free logics are the weakest of the three families considered above, i.e. they prove less. As the adding of ' $E$ ! $t$ ' to Specification and Particularization is the weakening factor for both kinds of free logics in the case of Lambert's square, so it is in the case of the Categories square, but only for positive free semantics. The following weakened versions are valid in the latter: 


$$
\begin{gathered}
P s \rightarrow(E ! \rightarrow \exists x(x=s)) \\
\sim \exists x(x=s) \rightarrow(E ! s \rightarrow \sim P s)
\end{gathered}
$$

A pattern involving existential premises is now easily recognizable. First of all, the amendment of the standard square of opposition - the inferences of which are strictly invalid in first-order logics that allow empty general terms - was made through the substitution of $\forall x P x \rightarrow P s$ and $\sim \exists x P x \rightarrow \exists x \sim P x$ by $\forall x P x \rightarrow(E ! s \rightarrow P s)$ and $\sim \exists x P x \rightarrow(E ! s \rightarrow \exists x \sim P x)$, respectively. Then Lambert's square was amended through the substitution of $\forall x P x \rightarrow P s$ and $\sim P s \rightarrow \exists x \sim P x$ by $\forall x P x$ $\rightarrow(E ! s \rightarrow P s)$ and $\sim P s \rightarrow(E ! \rightarrow \exists x \sim P x)$, respectively, thus generating free logic. Lastly, the Categories square, supported by classical predicate logic and negative free logics, was amended through the substitution of $P s \rightarrow \exists x(x=s)$ and $\sim \exists x(x=s) \rightarrow \sim P s$ by $P s \rightarrow(E ! \rightarrow \exists x(x=s))$ and $\sim \exists x(x=s) \rightarrow(E ! s \rightarrow \sim P s)$, respectively, thus making positive free logics able to support it. In all three cases, the introduction of $E$ ! $s$ between the original antecedents and consequents is what makes a logic able to support a given square, but also what makes it weaker

With respect to the issue of existential import, while the classical first-order reading of universal predications as universalized conditionals represented an improvement towards a solution to the problem, the analysis above shows, along with Lambert's, that free logics are powerful tools for clarifying the presuppositions underlying certain inferences that connect predications with general sentences.

\section{References}

1. Aristotle. Categories, Transl. by H. P. Cooke, Massachusetts: Harvard University Press, 1938.

2. Blanché, R. Structures Intellectuelles: essai sur l'organization systématique des concepts,Paris:J. Vrin, 2012.

3. Lambert, K. Free Logic and the Concept of Existence, Notre Dame Journal of Formal Logic, vol. 8, 1967, pp. 133-144.

4. Nolt, J. \& E.N. Zalta (eds.). Free Logic, In The Stanford Encyclopedia of Philosophy, 2014.

5. Russell, B. On Denoting, Mind, New Series, Vol. 14, No. 56, 1905, pp. 479-493.

6. Shang, F. \& S. Chatti. The Cube, the Square and the Problem of Existential Import, History and Philosophy of Logic, Vol. 34, No. 2, 2013, pp. 101-132.

\section{Notes}

1. The distinction inner/outer may seem to be an ad hoc way of making empty-termed sentences true, but it is possible to give it some philosophical account. While members of the inner domain represent "existing" objects, the members of the outer domain represent "non-existing" things like ideal entities, error objects, fictional objects or impossible objects [4]. This is the philosophical account for disjoint domains. If the convention makes the inner domain a subset of the outer domain, we can understand the distinction in Meinongian terms: while the members of the latter represent all objects that have being, the members of the former represent objects that have not only being, but are also "existing". In both cases, the only restriction is the non-emptiness of the outer domain.

2. The four relations of the logical square can be reduced to propositional truth-functions according to the following table [2, p. 29]:

\begin{tabular}{|l|l|l|l|}
\hline Relation & Truth-function & $\begin{array}{l}\text { Combinations of truth- } \\
\text { values }\end{array}$ & Symbol \\
\hline Contradiction & exclusive disjunction & VF; FV & w \\
\hline Contrariety & incompatibility & VF; FV; FF & I \\
\hline Subcontrariety & inclusive disjunction & VV; VF; FV & V \\
\hline Subalternation & Implication & VV; FV; FF & $\rightarrow$ \\
\hline
\end{tabular}


3. All of these can be replaced with formulae containing $n$-place predicates. We only use one-place predicates in order to better translate the sentences in the Categories square - Aristotle only considered sentences containing like "Socrates is sick" and "Socrates is well". 